\title{
Slow Peaking and Low-Gain Designs for Global Stabilization of Nonlinear Systems
}

\author{
Rodolphe Sepulchre, Member, IEEE
}

\begin{abstract}
This paper presents an analysis of the slow-peaking phenomenon, a pitfall of low-gain designs that imposes basic limitations to large regions of attraction in nonlinear control systems. The phenomenon is best understood on a chain of integrators perturbed by a vector field $u p(x, u)$ that satisfies $p(x, 0)=0$. Because small controls (or low-gain designs) are sufficient to stabilize the unperturbed chain of integrators, it may seem that smaller controls, which attenuate the perturbation $u p(x, u)$ in a larger compact set, can be employed to achieve larger regions of attraction. This intuition is false, however, and peaking may cause a loss of global controllability unless severe growth restrictions are imposed on $p(x, u)$. These growth restrictions are expressed as a higher order condition with respect to a particular weighted dilation related to the peaking exponents of the nominal system. When this higher order condition is satisfied, an explicit control law is derived that achieves global asymptotic stability of $x=0$. This stabilization result is extended to more general cascade nonlinear systems in which the perturbation $p(x, v) v, v=(\xi, u)^{T}$, contains the state $\xi$ and the control $u$ of a stabilizable subsystem $\dot{\xi}=a(\xi, u)$. As an illustration, a control law is derived that achieves global stabilization of the frictionless ball-and-beam model.
\end{abstract}

Index Terms-High gain, low gain, Lyapunov stabilization, nonlinear control.

\section{INTRODUCTION}

$\mathbf{H}$ IGH-GAIN and low-gain designs have served as cornerstones in the numerous developments in the semiglobal and global stabilization of nonlinear systems. They have been precursors to more flexible recursive Lyapunov designs [14], [13], [7], [15]; the simplest semiglobal recursive designs for nonlinear systems employ linear high-gain and low-gain control laws [22], [4], and the recent nonlinear generalizations of the small gain theorem have provided global versions of these designs [23], [3].

A proptotype of high-gain designs is for the scalar system $\dot{x}=x^{2}+u$, in which a higher gain $k \gg 1$ in the feedback $u=-k x$ allows us to further dominate the destabilizing nonlinearity $x^{2}$ and to further increase the region of attraction of the equilibrium $x=0$. A prototype of low-gain designs is for the scalar system $\dot{x}=u+u^{2}$, in which a lower gain $0<\epsilon \ll 1$ in the feedback $u=-\epsilon x$ allows us to further dominate the destabilizing nonlinearity $u^{2}$ and to further increase the region of attraction of the equilibrium $x=0$.

Early caveats in the literature-see, for instance, [9]—have shown that the intuition carried by these simple examples may

Manuscript received May 9, 1997; revised January 10, 1999. Recommended by Associate Editor, J.-B. Pomet.

The author is with the Institut Montefiore, University of Liège, 4000 Liège

Sart-Tilman, Belgium (e-mail: r.sepulchre@ulg.ac.be).

Publisher Item Identifier S 0018-9286(00)02143-7. fail in higher dimension. In the seminal paper [17], Sussmann and Kokotovic̀ exhibited the pitfall of the high-gain design — and in fact, a fundamental nonlinear limitation to achieving large regions of attractions - by considering the global stabilization of nonlinear cascades

$$
\begin{aligned}
\dot{x} & =f(x)+\psi(x, \xi) \xi \\
\dot{\xi} & =A \xi+b u
\end{aligned}
$$

where the equilibrium $x=0$ of $\dot{x}=f(x)$ is assumed to be globally asymptotically stable (GAS) and the pair $(A, b)$ is controllable. The simple high-gain intuition would suggest that the equilibrium $(x, \xi)=(0,0)$ of $(1.1)$ is always semiglobally stabilizable: a faster stabilization of $\xi(t)$ causes a faster convergence to zero of the interconnection term $\psi(x, \xi) \xi$, which perturbs the GAS system $\dot{x}=f(x)$. This reasoning is false, however. As an illustration, an analysis in [15, p. 167] shows that the system

$$
\begin{aligned}
\dot{x} & =-x+x^{2}\left(\dot{\xi}_{1}^{2}+\xi_{2}^{2}\right) \\
\dot{\xi}_{1} & =\xi_{2} \\
\dot{\xi}_{2} & =u
\end{aligned}
$$

is not semiglobally stabilizable and that, for large initial conditions, no open-loop control $u(t)$ exists that drives the solution to $(x, \xi)=(0,0)$. In this case, the failure of the high-gain intuition is caused by the (fast) peaking phenomenon inherent to any high-gain design: a fast stabilization of $\xi_{1}(t)\left[\right.$ say, $\xi_{1}(t) \approx$ $e^{-k t}$ ] causes a large peaking of its derivative $\xi_{2}(t)=\dot{\xi}_{1}(t)$ $\left(\approx k e^{-k i t}\right)$. In the example (1.2), the fast stabilization of $\xi_{1}$ is necessary to increase the region of attraction, but the peaking of $\xi_{2}(t)$ causes the finite escape time of $x(t)$ to infinity.

To overcome the effect of peaking in the stabilization of the cascade (1.1), it is necessary to restrict the form of the interconnection $\psi(x, \xi) \xi$ either by structural requirements (only the "nonpeaking" states of the $\xi$-subsystem appear in the interconnection) or by growth conditions on the $x$-subsystem to prevent the possibility of finite escape time. Structural requirements led to the developement of recursive design procedures for "strict-feedback" systems [14], [10], and the absence of finite escape time for $x(t)$ can be guaranteed by input-to-state stability conditions [18] or growth restrictions [17], [14], [15]. Limitations to semiglobal stabilization because of (fast) peaking are further analyzed in [2] and [15].

Low-gain designs attracted many researchers in the recent years with the work by Lin and Saberi [11] and Teel [19], who showed that a chain of integrators (and, more generally, linear systems having all their eigenvalues in the closed left-half plane [18]) can be stabilized by a control law bounded by an arbitrarily small constant. In this paper, we show that the low-gain 
intuition has a pitfall similar to the high-gain intuition. We will exhibit this limitation of low-gain designs by considering the global asymptotic stabilization of a perturbed chain of integrators

$$
\left(\begin{array}{c}
\dot{x}_{1} \\
\vdots \\
\dot{x}_{n-1} \\
\dot{x}_{n}
\end{array}\right)=\left(\begin{array}{c}
x_{2} \\
\vdots \\
x_{n} \\
u
\end{array}\right)+u p(x, u), \quad p(x, 0)=0 .
$$

Because the (unperturbed) chain of integrators can be globally stabilized by a low-gain design, the perturbation $u p(x, u)$ can be rendered arbitrarily small in any compact set. More precisely, in any given compact set, the bound $\epsilon$ on the control that achieves global stabilization of the chain of integrators can be selected small enough such that $u\|p(x, u)\|=O\left(\epsilon^{2}\right)$ within this compact set. The scalar low-gain intuition would then suggest that the size of the region of attraction for the perturbed system (1.3) can be arbitrarily increased by diminishing the bound $\epsilon$ on the control law.

This reasoning is also false, however. A calculation in Section II shows that the system

$$
\begin{aligned}
& \dot{x}_{1}=x_{2} \\
& \dot{x}_{2}=u+x_{1}^{\nu} u^{2}
\end{aligned}
$$

is globally stabilizable if $\nu=0$ or $\nu=1$. This system, however, is not even semiglobally stabilizable if $\nu>1$. As in the example (1.2), for large initial conditions, no open-loop control $u(t)$ exists that drives the solution to $x=0$. We show in Section II that this failure of the low-gain intuition in the system (1.4) is caused by the (slow) peaking phenomenon inherent to any low-gain design: a slow stabilization of $x_{2}(t)$ [say, $\left.x_{2}(t) \approx e^{-\epsilon t}\right]$ implies a large peaking of its integral $x_{1}(t)=\int x_{2}(t)\left(\approx(1 / \epsilon) e^{-\epsilon t}\right)$. In the example (1.4), the peaking of $x_{1}(t)$ causes the perturbation $x_{1}^{\nu} u^{2}$ to dominate $u$ if $\nu>1$. If a chain of integrators is stabilized by using a low-gain design, all states, except for $x_{n}$, undergo a large transient for certain initial conditions. A smaller bound on the control law causes larger transients that may amplify the effect of the perturbation $p(x, u)$ and prevent large regions of attractions. The peaking associated with low-gain designs occurs in the slow time scale $\tau=\epsilon t$. It is a dual phenomenon to the peaking associated with high-gain designs, which occurs in the fast time scale $\tau=(t / \epsilon)$.

To achieve large regions of attraction for the system (1.3) with a low-gain design, we must restrict the form of the perturbation $p(x, u) u$ by either imposing structural requirements [an upper triangular structure for $p(x, u)]$ or by restricting the growth of the nonlinearities. Restricting the structure of $p(x, u)$, Lin and Saberi [12] and Teel [21] obtained results in the case of pure input nonlinearities; that is, $p(x, u)=p(u)$. This process led to subsequent recursive designs for feedforward systems [20], [13], [7]. In this paper, we will analyze the growth restrictions that must be imposed on $p(x, u) u$ to guarantee large regions of attraction in the absence of structural restrictions.

By weighting the growth of the nonlinearities with the peaking exponent of the different states, we will show that the growth condition that guarantees large regions of attractions can be expressed as a higher order condition on the vector field $p(x, u)$ with respect to a particular weighted dilation. When this higher order condition is not satisfied, the slow-peaking phenomenon may prevent semiglobal stabilization. On the contrary, when the higher order condition holds, we achieve global stabilization with an explicit control law. Like several low-gain designs previously proposed in the literature, our control law is obtained in the form of a sum of saturations. A distinct feature of our design is that the saturation levels are state dependent rather than constant. They are selected in such a way that the higher order property of the perturbation $u p(x, u)$ is preserved in closed loop.

In the last section of the paper, our analysis of the system (1.3) is extended to nonlinear cascades of the form

$$
\begin{aligned}
\left(\begin{array}{c}
\dot{x}_{1} \\
\vdots \\
\dot{x}_{n-1} \\
\dot{x}_{n}
\end{array}\right) & =\left(\begin{array}{c}
x_{2} \\
\vdots \\
x_{n} \\
c^{T} v
\end{array}\right)+P(x, v) v, \quad P(x, 0)=0 \\
\dot{\xi} & =a(\xi, u) \quad \xi \in \mathbb{R}^{p}, u \in \mathbb{R}, v=(\xi, u)^{T}
\end{aligned}
$$

where the equilibrium $\xi=0$ of $\dot{\xi}=a(\xi, 0)$ is assumed to be GAS and LES. As an illustration, we show that the popular model of the frictionless ball-and-beam model, which does not meet the structural requirements of any recursive design previously proposed in the literature, satisfies the growth restrictions of this paper and can be globally stabilized by a low-gain design. Global stabilization of the (frictionless) ball-and-beam was first proposed in [1], where a similar low-gain control law is employed.

The paper is organized as follows. In Section II, we describe the slow-peaking phenomenon associated with low-gain designs and the obstacle that it constitutes to achieve large regions of attractions. In Section III, we characterize the absence of peaking as a "higher order" property of the perturbation with respect to a weighted dilation. Section IV is devoted to the global stabilization result for the system (1.3) under the suitable growth restriction for $p(x, u) u$. This stabilization result is extended to the cascade (1.5) in Section V.

\section{Low-Gain Designs AND Slow PeaKing}

Throughout this paper, we call a low-gain design a control law $u=k(x)$ that achieves global stabilization of the equilibrium $x=0$ in such a way that, for any $\epsilon>0$, the parameters of the control law can be tuned to satisfy

$$
\sup _{t \geq 0}|u(x(t))| \leq \gamma(\|x(0)\|) \epsilon
$$

for some continuous, positive function $\gamma$ independent of $\epsilon$.

For a chain of integrators, a linear low-gain design is obtained by scaling the eigenvalues to be sufficiently slow [11]. Nonlinear low-gain designs allow the bound on the control law to be independent of the initial condition. They typically make use of the saturation function $\operatorname{sat}_{M}(s)$, which is linear close to the origin, say, $\operatorname{sat}_{M}(s)=s$, and then saturated at a constant $M=0(\epsilon)$. Nonlinear low-gain designs for the chain of integrators have been proposed in the form of a nested saturation scheme [19]

$$
u=-\operatorname{sat}_{M_{n}}\left(y_{n}+\operatorname{sat}_{M_{n-1}}\left(y_{n-1}+\cdots+\operatorname{sat}_{M_{1}}\left(y_{1}\right)\right) \cdots\right)
$$


where $y_{i}=\sum_{k=i}^{n} x_{k}$, or in the form of a sum of saturations [18]

$$
u=-\operatorname{sat}_{M_{n}}\left(y_{n}\right)-\operatorname{sat}_{M_{n-1}}\left(y_{n-1}\right)-\cdots-\operatorname{sat}_{M_{1}}\left(y_{1}\right) \text {. }
$$

Even though low-gain designs achieve global stabilization for any value of the small parameter $\epsilon>0$, a variation of $\epsilon$ significantly affects the transient behavior of the solutions. As $\epsilon \rightarrow 0$, not only the convergence of the solutions is slowed, but also certain states undergo large transients. The simplest illustration of this fact is the second-order system $\dot{x}_{1}=x_{2}, \dot{x}_{2}=u$, for which the low-gain design $-2 \epsilon x_{2}-\epsilon^{2} x_{1}$ places the two poles at $\lambda=-\epsilon$. If $x_{1}(0)=0$, the solution is

$$
x_{1}(t)=x_{2}(0) t e^{-\epsilon t}, \quad x_{2}(t)=x_{2}(0)(1-\epsilon t) e^{-\epsilon t .}
$$

The state $x_{1}$ attains its maximum value $\bar{x}_{1}=\left(e^{-1} x_{2}(0)\right) / \epsilon$ at time $t=1 / \epsilon$. This peaking of the state $x_{1}$ is proportional to $1 / \epsilon$ and occurs in the slow time scale $\tau=\epsilon t$. The following proposition shows that this phenomenon is inherent to any low-gain design, that is, a consequence of the bound (2.6), and that the peaking is worse for states located farther from the input.

Proposition 1 [15]: Define the peaking exponent of the variable $x_{i}$ as the smallest integer $\pi$ for which the following bound holds:

$$
\sup _{t \geq 0}\left|x_{i}(t)\right| \leq \gamma(\|x(0)\|) \epsilon^{-\pi}
$$

Then, every low-gain design for the chain of integrators that satisfies the bound (2.6) causes the state $\xi_{i}$ to peak with an exponent $\pi=n-i$ for $i \in\{1, \cdots, n\}$.

Slow peaking imposes restrictions on the nonlinearities in system (1.3). For instance, a low-gain design is necessary to achieve large regions of attraction for the system

$$
\begin{aligned}
& \dot{x}_{1}=x_{2} \\
& \dot{x}_{2}=u+x_{1}^{\nu} u^{2}
\end{aligned}
$$

because the control $u$ must dominate the perturbation $x_{1}^{\nu} u^{2}$. A smaller bound $\epsilon$ on the control law, however, will cause a larger peak $0(1 / \epsilon)$ for the state $x_{1}$. When $x_{1}$ reaches its peak, the perturbation $x_{1}^{\nu} u^{2}$ is $0\left(\epsilon^{2-\nu}\right)$. If $\nu>1$, the following calculation shows that semiglobal stabilization is impossible.

Let $x_{1}(0)=1$ and $x_{2}(0)>1$ so that $x_{1}(t) \geq 1+t$ as long as $x_{2}(t)>1$. When $x_{1}>0$, the minimum value for $\dot{x}_{2}$ is obtained with the control $u=-\left(1 / 2 x_{1}^{\nu}\right)$, which yields

$$
\dot{x}_{2}(t) \geq-\frac{1}{4 x_{1}(t)^{\nu}} \text {. }
$$

By integration, this process implies [as long as $x_{2}(t)>1$ ]

$$
\begin{aligned}
x_{2}(t) & \geq x_{2}(0)-\int_{0}^{t} \frac{d s}{4(1+s)^{\nu}} \\
& \geq x_{2}(0)-\int_{0}^{\infty} \frac{d s}{4(1+s)^{\nu}} .
\end{aligned}
$$

For $\nu>1$, the last integral is bounded by a constant $K$. Choosing $x_{2}(0)>K+1$, we conclude that $x_{2}(t) \geq 1$ for all $t \geq 0$, which shows that semiglobal stabilization is impossible.

\section{GROWTH CONDITIONS AND WeIGHTED HoMOGENEITY}

Because of peaking, growth restrictions must be imposed on $p(x, u)$ to achieve large regions of attractions in the system
(1.3). Because different states have different peaking exponents, the growth restrictions will be different with respect to the different states and for each component $p_{i}(x, u)$. To express such growth restrictions in a general framework, we will employ the notion of weighted homogeneity for (scalar) functions and vector fields [8], [6]. These tools are convenient to define growth conditions, not in terms of the usual norm $\|x\|$, but in terms of a norm that weights each state by its peaking exponent.

The Slow-Peaking Dilation: A dilation $\delta_{\epsilon}^{r}$ is a map $\delta_{\epsilon}^{r}: \mathbb{R}^{n} \rightarrow$ $\mathbb{R}^{n}: \delta_{\epsilon}^{r}(x)=\left(\epsilon^{r_{1}} x_{1}, \cdots, \epsilon^{r_{n}} x_{n}\right)$ with $\epsilon>0$. The integers $r_{i}$ are called the weights of the dilation. In this paper, we use both positive and negative weights, which is not standard in the literature. Classical homogeneity notions are defined for the standard dilation, that is, when all $r_{i}=1$. In the more general framework of [6] and [8], different weights are used, but they satisfy the condition $r_{n} \geq r_{n-1} \geq \cdots \geq r_{1} \geq 1$. Here, we want to weight the state $x_{i}$ of the chain of integrators by its peaking exponent $n-i$, which motivates the dilation

$$
\delta_{\epsilon}(x)=\left(\epsilon^{1-n} x_{1}, \cdots, \epsilon^{i-n} x_{i}, \cdots, \epsilon^{0} x_{n}\right) .
$$

Homogeneous Functions and Order: A continuous (scalar) function $h(x)$ is said to be homogeneous of degree $r$ with respect to the dilation $\delta_{\epsilon}$ if it satisfies $h\left(\delta_{\epsilon}(x)\right)=\epsilon^{r} h(x)$. For instance, the monomial $h(x)=x_{i}^{p}$ is homogeneous of degree $p$ with respect to the standard dilation, but is homogeneous of degree $(i-n) p$ with respect to the weighted dilation (3.12).

A function $h: \mathbb{R}^{n} \rightarrow \mathbb{R}$ is classically said to be of order $\nu$ if $|h(x)| \leq C\|x\|^{\nu}$ as $\|x\| \rightarrow 0$, which can be rephrased as the condition

$$
\frac{\left|h\left(\epsilon x_{1}, \cdots, \epsilon x_{n}\right)\right|}{\epsilon^{\nu}} \leq C \quad \text { as } \epsilon \rightarrow 0 \text { and }\|x\|=1 .
$$

A notion of order is similarly attached to any dilation if we replace $\left|h\left(\epsilon x_{1}, \cdots, \epsilon x_{n}\right)\right|$ by $h\left(\delta_{\epsilon}(x)\right)$ in the above condition. In the case of the standard dilation, the order property characterizes a property of the function in the neighborhood of $x=0$ because $\lim _{\epsilon \rightarrow 0} \delta_{\epsilon}(x)=0$. In the case of a general dilation, the order property characterizes a property of the function in a region of $\mathbb{R}^{n}$, which depends on the sign of the different weights. If the weight associated to $x_{i}$ is positive, the order condition is a property of the function for $\left|x_{i}\right|$ small. On the contrary, if the weight is negative, the order condition is a property of the function for $\left|x_{i}\right|$ large. If the weight is zero, the order condition does not restrict the dependence of $h$ on the variable $x_{i}$. For the dilation (3.12), all weights are negative, except the zero weight on $x_{n}$. In this case, the order condition expresses a property for $\left\|\left(x_{1}, \cdots, x_{n-1}\right)\right\|$ large, but this property can be nonuniform in $x_{n}$ : we say that a function $h: \mathbb{R}^{n} \rightarrow \mathbb{R}$ is of order $\nu$ with respect to the dilation (3.12) if for each $x_{n} \in \mathbb{R}$, and for a fixed constant $\Delta>0$, the following holds:

$$
\left\|\left(x_{1}, \cdots, x_{n-1}\right)\right\| \geq \Delta \Rightarrow \lim _{\epsilon \rightarrow 0} \frac{\mid h\left(\delta_{\epsilon}(x) \mid\right.}{\epsilon^{\nu}} \leq \gamma\left(x_{n}\right)
$$

where $\gamma\left(x_{n}\right)>0$ is continuous in $\mathbb{R}$ [ the choice of $\Delta>0$ is arbitrary, but in general, $\gamma(\cdot)$ will depend on $\Delta]$. 
The positive function

$$
\rho(x)=\left(1+\sum_{\substack{i=1 \\ \alpha_{i}>0}}^{n-1} \alpha_{i} x_{i}^{(2(n-1)) /(n-i)}\right)^{-(1 /(2(n-1)))}
$$

defines a "radius" of order one with respect to the dilation (3.12), with the property that, for $1 \leq i \leq n-1$, the variable $z_{i}=$ $\rho^{n-i}(x) x_{i}$ is uniformly bounded in $\mathbb{R}^{n}$. For notational convenience, we note $z_{n}=x_{n}$.

To extend the homogeneity definitions to a function that depends on the control, we consider the extended dilation

$$
\delta_{\epsilon}(x, u)=\left(\epsilon^{1-n} x_{1}, \cdots, \epsilon^{-1} x_{n-1}, x_{n}, \epsilon u\right) .
$$

A continuous (scalar) function $h(x, u)$ is homogeneous of degree $r$ if $h\left(\delta_{\epsilon}(x, u)\right)=\epsilon^{r} h(x, u)$. It is of order $r$ if for each $x_{n}$, and for a fixed constant $\Delta>0$, it satisfies

$$
\left\|\left(x_{1}, \cdots, x_{n-1}\right)\right\| \geq \Delta \Rightarrow \lim _{\epsilon \rightarrow 0} \frac{\left|h\left(\delta_{\epsilon}(x, u)\right)\right|}{\epsilon^{r}} \leq \gamma\left(x_{n}\right)
$$

where $\gamma\left(x_{n}\right)>0$ is continuous in $\mathbb{R}$. The following proposition is a consequence of the above definitions and allows us to reformulate the order condition in terms of the weighted radius (3.13).

Proposition 2: Consider the radius defined by (3.13).

1) If $h(x)$ is a continuous function of order $\nu$, then a positive function $C\left(x_{n}\right)>0$ exists such that

$$
|h(x)| \leq C\left(x_{n}\right) \rho^{\nu}(x) .
$$

2) If $h(x, u)$ is a differentiable function of order $\nu$ and $h(x, 0)=0$, then, for every $r \geq 1$ and $M>0$, we have

$$
\begin{aligned}
|u| & \leq M \rho^{r}(x) \Rightarrow|h(x, u)| \\
& \leq M C\left(x_{n}\right) \rho^{\nu+r-1}(x) .
\end{aligned}
$$

Proof:

1) Because $z_{i}=\rho^{n-i} x_{i}$ and $\left(\delta_{\rho}(x)\right)_{i}=\rho^{i-n} x_{i}$, we have

$$
\forall x \in \mathbb{R}^{n}: \quad h(x)=h\left(\delta_{\rho(x)}(z)\right) .
$$

If $h$ is of order one, this implies that a sufficiently small $\bar{\epsilon}>0$ exists such that $|h(x)| \leq C\left(x_{n}\right) \rho^{\nu}(x)$ for all $x \in\{x: \rho(x) \leq \bar{\epsilon}\}$. On the other hand, for each $x_{n}$, the set in which $\rho(x) \geq \bar{\epsilon}$ is compact, so that the constant

$$
K\left(x_{n}\right)=\max _{\rho(x) \geq \bar{\epsilon}} \frac{|h(x)|}{\bar{\epsilon}^{\nu}}
$$

is finite. The continuous function $C\left(x_{n}\right)$ in (3.15) can thus be selected to dominate $\max \left(K\left(x_{n}\right), \gamma\left(x_{n}\right)\right)$.

2) Because $h(x, 0)=0$ and $h$ is differentiable, we have $h(x, u)=u \widetilde{h}(x, u)$, where $\tilde{h}(x, u)$ is a continuous function of order $\nu-1$. Applying the same argument as in $1)$, we conclude that $|\tilde{h}(x, u)| \leq C\left(x_{n}\right) \rho^{\nu-1}(x)$ for $\left|u \rho^{-1}(x)\right| \leq M$. Multiplying both sides by $u$ and using the fact that $|u| \leq M \rho^{r}(x)$ (with $r \geq 1$ ), we obtain

$$
h(x, u)=u \tilde{h}(x, u) \leq M \rho^{r}(x) C\left(x_{n}\right) \rho^{\nu-1}(x)
$$

which proves the claim.

In the following, the expression $h(x)=0\left(\rho^{\nu}(x)\right)$ will denote a continuous function of order $\nu$, that is, a function that satisfies (3.15) for some positive function $C\left(x_{n}\right)>0$.
Homogeneous Vector Field: A vector field $f: \mathbb{R}^{n} \rightarrow \mathbb{R}^{n}$ is homogeneous of degree $\tau$ if $L_{f} h(x)$ is homogeneous of degree $r+\tau$ whenever $h(x)$ is homogeneous of degree $r$. It is of order $\tau$ if $L_{f} h$ is of order $r+\tau$ whenever $h$ is of order $r$. The (controlled) vector field $f(x, u)$ is homogeneous of degree $\tau$ if $L_{f} h(x, u)$ is homogeneous of degree $r+\tau$ whenever $h(x)$ is homogeneous of degree $r$. It is of order $\tau$ if $L_{f} h$ is of order $r+\tau$ whenever $h$ is of order $r$.

To check that $f(x)$ is a vector field of order $\nu$ with respect to (3.12), we only need to verify that the $i$ th component $f_{i}(x)$ is a function of order $i-n+\nu$. For instance, the chain of integrators $\dot{x}_{1}=x_{2}, \cdots, \dot{x}_{n}=u$ is homogeneous of degree one because $f_{i}(x)=x_{i+1}$ is homogeneous of degree $i+1-n$ for $1 \leq i \leq$ $n-1$ and $f_{n}(x)=u$ is homogeneous of degree 1 . Finally, as a consequence of Proposition 2, a controlled vector field $f(x, u)$ that satisfies $f(x, 0)=0$ is of the same order as the closed-loop vector field $f(x, k(x))$ provided that the control law $u=k(x)$ is a function of order one.

Growth Restrictions as a Higher Order Condition: We have just seen that the chain of integrators is of order one with respect to the slow-peaking dilation (3.14). In system (2.10), $u p(x, u)=\left(0, x_{1}^{\nu} u^{2}\right)$ is homogeneous of degree $2-\nu$. If $\nu>1$, the order of the perturbation $u p(x, u)$ is lower than the order of the chain of integrators, and we have seen that semiglobal stabilization is impossible in this case. The higher order property with respect to the slow-peaking dilation (3.14) thus characterizes the maximal admissible growth of the vector field $u p(x, u)$ to guarantee that the slow peaking of the variables is not an obstacle to large regions of attraction.

\section{A Low-Gain Design FOR Global Stabilization}

Motivated by our analysis of peaking, we will achieve global stabilization of the system (1.3) under the following growth assumption.

Assumption 1: The vector field $p(x, u) u$ is at least of order one with respect to the dilation (3.14).

The higher order property of the perturbation $u p(x, u)$ guaranteed by Assumption 1 will be preserved in closed loop by designing a low-gain control law of order one. To this end, we will start from the low-gain control law (2.8), which achieves global stabilization of the chain of integrators, but we will make the saturation constants of the control law state dependent: precisely, we will employ the control law

$$
\begin{aligned}
u= & -\operatorname{sat}_{M \epsilon(x)}\left(y_{n}\right)-\operatorname{sat}_{M \epsilon^{2}(x)}\left(y_{n-1}\right) \\
& -\cdots-\operatorname{sat}_{M \epsilon^{n}(x)}\left(y_{1}\right), \quad 0<M \leq 1
\end{aligned}
$$

where the low gain $\epsilon(x)$ is of order one with respect to the dilation (3.12)

$$
\epsilon(x)=\min \left(\bar{\epsilon}\left(x_{n}\right), \rho(x)\right), \quad 0<\bar{\epsilon}\left(x_{n}\right)<1 .
$$

The control law (4.17) is of order one, and we will show that it achieves global asymptotic stabilization of the chain of integrators. Our proof follows the same lines as Teel's proof for the saturation design (2.7). We will show that, for each solution of the closed-loop system, a sequence of instants exists $T_{n} \geq T_{n-1} \geq \cdots \geq T_{1}$ such that $\operatorname{sat}_{M \epsilon^{n-i+1}(x)}\left(y_{i}\right)=y_{i}$ for all $t \geq T_{i}$. After the finite time $T_{1}$, the control law is linear and the convergence of the solutions is exponential. 
Because the saturations in (4.18) are state dependent and tend to zero as $\left\|\left(x_{1}, \cdots, x_{n-1}\right)\right\| \rightarrow \infty$, the analysis faces two difficulties not present in the constant saturation scheme (2.7). These difficulties are easily illustrated by the scalar system

$$
\dot{z}=-\operatorname{sat}_{M(t)}(z), \quad z \in \mathbb{R} .
$$

Global asymptotic stability of the equilibrium $z=0$ of (4.19) requires that $M(t)$ is not integrable; that is

$$
\forall t \geq 0: \quad \lim _{T \rightarrow \infty} \int_{t}^{t+T} M(s) d s=+\infty .
$$

A similar nonintegrability condition will be necessary for the time function $M \epsilon^{i}(x(t))$ evaluated along the solutions of the system (1.3).

Under the condition (4.20), a finite time $T \geq t_{0}$ exists such that $\operatorname{sat}_{M(T)}(z(T)) \leq z(T)$; that is, the linear zone of the saturation is attained. The slowly varying condition

$$
\dot{M}(t) \leq \mu M(t), \quad \mu \in(0,1)
$$

ensures that $\operatorname{sat}_{M(t)}(z(t))$ is no longer saturated for all future times because

$$
\begin{aligned}
& \left.\frac{d}{d t}(|z(t)|-M(t))\right|_{|z(t)|=M(t)} \\
& \quad=-M(t)-\dot{M}(t) \leq-(1-\mu) M(t)<0 .
\end{aligned}
$$

The analog of the properties (4.20) and (4.21) required for the saturation design (4.18) are given in the following lemmas, proven in the Appendix.

Lemma 1: Consider the system (1.3) with a control law $u=$ $k(x)$ of order $(n-i+1)$ for some $i \in\{1, \cdots, n\}$. Assume that $p(x, u)$ is of order zero. Then, for each solution $x(t)$ whose last $(n-i+1)$ components are uniformly bounded [that is, $\left|x_{k}(t)\right| \leq \bar{x}_{k}$ for all $t \geq 0$ and for $\left.i \leq k \leq n\right]$, the following holds:

$$
\lim _{t \rightarrow \infty} \int_{0}^{t} \rho(x(s))^{n-i+1} d s=+\infty .
$$

Lemma 2: Let $f(x, u)$ be a vector field of order one, and let $u(t)$ be a control that satisfies at each time $|u(t)| \leq K_{u} \epsilon(x(t))$. Then, the time derivative of the low-gain (4.18) along the solution of $\dot{x}=f(x, u)$ satisfies

$$
|\dot{\epsilon}(x)|<\mu \epsilon(x)
$$

where the constant $\mu=\mu(\bar{\epsilon})>0$ can be rendered arbitrary small by a suitable choice of $\bar{\epsilon}\left(x_{n}\right)$.

We now prove that the control law (4.17), with $M=1$, and a constant $\bar{\epsilon}\left(x_{n}\right)$, achieves global stabilization of the chain of integrators.

Proposition 3: The low-gain control law (4.17) with $M=1$ and $\bar{\epsilon}\left(x_{n}\right)=\bar{\epsilon}>0$ achieves global asymptotic stability of the equilibrium $x=0$ of the (unperturbed) chain of integrators provided that $\bar{\epsilon}$ is sufficiently small.

Proof: The Jacobian linearization of the closed-loop system is Hurwitz, and the equilibrium $x=0$ is therefore locally exponentially stable. Hence, we only show that, for an arbitrary initial condition $x(0) \in \mathbb{R}^{n}$, the solution $x(t)$ asymptotically converges to $x=0$. The last equation of the closed-loop system satisfies

$$
\left|x_{n}\right| \geq \epsilon(x) \Rightarrow \dot{x}_{n}=-\operatorname{sign}\left(x_{n}\right) \epsilon(x)+0\left(\epsilon(x)^{2}\right)
$$

so that $\left|x_{n}(t)\right|$ is bounded by $\max \left(\left|x_{n}(0)\right|, \bar{\epsilon}\right)$. By integration on an interval $[0, T]$, we obtain

$$
\begin{aligned}
\left|x_{n}(t)\right| & \geq \epsilon(x(t)) \quad \forall t \in[0, T] \Rightarrow\left|x_{n}(T)\right| \\
& \leq\left|x_{n}(0)\right|-\frac{1}{2} \int_{0}^{T} \epsilon(x(t)) d t .
\end{aligned}
$$

Applying Lemma 1 with $i=n$, we have that $\epsilon(x(t))$ is not integrable over $[0, \infty)$. Because the right-hand side of (4.24) cannot become negative, we conclude that a finite time $T_{n}>0$ exists such that $\left|x_{n}\left(T_{n}\right)\right| \leq \epsilon\left(x\left(T_{n}\right)\right)$. On the other hand, the time derivative of $\left|x_{n}(t)\right|-\epsilon(x(t))$ satisfies

$$
\begin{aligned}
& \left.\frac{d}{d t}\left(\left|x_{n}\right|-\epsilon(x)\right)\right|_{\left|x_{n}\right|=\epsilon(x)} \\
& \quad=-\epsilon(x)+0\left(\epsilon^{2}\right)-\dot{\epsilon} \leq-(1-\mu) \epsilon(x)+0\left(\epsilon^{2}\right)<0
\end{aligned}
$$

where the inequalities hold provided that $\mu$ is sufficiently small. We conclude that $\left|x_{n}(t)\right| \leq \epsilon(x(t))$ for all $t \geq T_{n}$, so that, for $t \geq T_{n}$, we have $\dot{x}_{n}=-x_{n}+0\left(\epsilon^{2}\right)$. The time derivative of $y_{n-1}=x_{n-1}+x_{n}$ then satisfies

$$
\left|y_{n-1}\right| \geq \epsilon^{2}(x) \Rightarrow \dot{y}_{n-1}=-\operatorname{sign}\left(y_{n-1}\right) \epsilon^{2}(x)+0\left(\epsilon(x)^{3}\right)
$$

so that $y_{n-1}(t)$ and $x_{n}(t)$ are bounded for all $t \geq 0$. Applying Lemma 1 with $i=n-1$, we conclude that $\epsilon^{2}(x(t))$ is not in $L^{1}[0, \infty)$ and pursue the proof as before to conclude that a finite time $T_{n-1} \geq T_{n}$ exists such that, for all $t \geq T_{n-1}$, we have $\left|y_{n-1}(t)\right| \leq \epsilon^{2}(t)$. Repeating the argument for each equation proves that, after a finite time $T_{1} \geq T_{2} \geq \cdots \geq T_{n}$, the control law is no longer saturated; that is, the solution exponentially converges to the origin.

The multiplication of the control law (4.17) by a gain $\gamma\left(x_{n}\right)>0$ does not change its order property. This only modification in the control law allows us to extend the result of Proposition 3 to the system (1.3), with the help of the following lemma.

Lemma 3: Consider the system $\dot{y}=A y+e(t)$, where $A$ is Hurwitz and the vector $e(t)$ is bounded by a slowly time-varying function; that is

$$
\|e(t)\| \leq K \epsilon(t), \quad|\dot{\epsilon}(t)|<\mu \epsilon(t) .
$$

Then, for $\mu>0$ sufficiently small, a constant $C>0$ exists such that each solution satisfies after a finite time $T>0$

$$
\forall t \geq T: \quad\|y(t)\| \leq C \epsilon(t) .
$$

[The result is obviously unchanged if $A$ is replaced by $A+E(t)$, where $E(t)$ is a perturbation satisfying $\|E(t)\|=0(M)$ for a sufficiently small constant $M$.]

We are now ready to state the main result of this section.

Theorem 1: If Assumption 1 holds, then a low-gain $\gamma\left(x_{n}\right)$ exists, a constant $M>0$ and a constant $\delta>0$ such that for all $M \in(0, \bar{M}]$, and (constant) $\bar{\epsilon} \in(0, \delta]$, the low-gain control law

$$
\begin{aligned}
u= & k(x)=-\gamma\left(x_{n}\right)\left(\operatorname{sat}_{M \epsilon(x)}\left(y_{n}\right)\right. \\
& \left.+\operatorname{sat}_{M \epsilon^{2}(x)} \cdots+\operatorname{sat}_{M \epsilon^{n}(x)}\left(y_{1}\right)\right)
\end{aligned}
$$

achieves global asymptotic stability of the equilibrium $x=0$ of system (1.3).

Proof: The last equation of the closed-loop system is $\dot{x}_{n}=-\gamma_{n}\left(x_{n}\right)\left(\operatorname{sat}_{M \epsilon(x)}\left(y_{n}\right)+0\left(M \epsilon^{2}\right)\right)\left(1+p_{n}(x, k(x))\right.$. 
Because $|k(x)| \leq M \gamma\left(x_{n}\right) \rho(x)$, Proposition 2 implies the existence of a positive function $C\left(x_{n}\right)>0$ such that

$$
\left|p_{n}(x, k(x))\right| \leq M \gamma\left(x_{n}\right) C\left(x_{n}\right) .
$$

We can choose the gain $\gamma\left(x_{n}\right)$ in such a way that $\gamma\left(x_{n}\right) \equiv 1$ for $\left|x_{n}\right| \leq 1$ and the product $\gamma\left(x_{n}\right) C\left(x_{n}\right)$ is uniformly bounded by a constant $C>0$. With such a gain, $\left|p_{n}(x, k(x))\right|=0(M)$, and for $M$ sufficiently small, the same argument as in Proposition 3 can be used to prove that a finite time $T_{n}>0$ exists such that, for all $t \geq T_{n},\left|x_{n}(t)\right| \leq M \epsilon(x(t))$. The time derivative of $x_{n}$ then becomes

$$
\dot{x}_{n}=-\left(x_{n}+0\left(M \epsilon^{2}\right)\right)(1+0(M)) .
$$

By Lemma $2,\left|(d / d t) \epsilon(x)^{2}\right| \leq 2 \mu \epsilon(x)^{2}$, where $\mu$ can be rendered arbitrarily small by decreasing $\bar{\epsilon}$. Lemma 3 can thus be applied to the system (4.27) to conclude that, after a finite time $T_{n}^{\prime} \geq T_{n}$, we have

$$
\forall t \geq T_{n}^{\prime}: \quad\left|x_{n}(t)\right|=0\left(M \epsilon(x)^{2}\right) .
$$

For $t \geq T_{n}^{\prime}$, the time derivative of $y_{n-1}=x_{n-1}+x_{n}$ satisfies

$$
\begin{aligned}
\dot{y}_{n-1}= & -\left(\operatorname{sat}_{M \epsilon(x)^{2}}\left(y_{n-1}\right)+0\left(M \epsilon(x)^{3}\right)\right)(1+0(M)) \\
& +x_{n} 0(M)+k(x) p_{n-1}(x, k(x))
\end{aligned}
$$

and the control law becomes of order two

$$
\begin{aligned}
|k(x)| & \leq\left|x_{n}\right|+\left|\operatorname{sat} M \epsilon(x)^{2}\left(y_{n-1}\right)+0\left(M \epsilon(x)^{3}\right)\right| \\
& \leq C M \rho^{2}(x) .
\end{aligned}
$$

By Proposition 2, $\left\|p_{n-1}(x, k(x))\right\|=0(M)$ so that the last term in (4.28) is $0\left(M^{2} \epsilon^{2}\right)$. So is the term $x_{n} 0(M)$, so that we can rewrite (4.28) as

$$
\dot{y}_{n-1}=-\operatorname{sat}_{M \epsilon(x)^{2}}\left(y_{n-1}\right)+0\left(M^{2} \epsilon(x)^{2}\right) .
$$

This process implies that $\left|y_{n-1}(t)\right|$ is bounded, and, proceeding as in the proof of Proposition 3, that a finite time $T_{n-1} \geq$ $T_{n}^{\prime}$ exists such that, for all $t \geq T_{n-1}$, we have $\left\|y_{n-1}(t)\right\| \leq$ $\left|M \epsilon(x(t))^{2}\right|$. For $t \geq T_{n-1}$, we rearrange the two last equations of the closed-loop system in the form

$$
\begin{aligned}
\left(\begin{array}{c}
\dot{y}_{n-1} \\
\dot{y}_{n}
\end{array}\right)= & \left(\begin{array}{cc}
-1+0(M) & 0(M) \\
-1+0(M) & -1+0(M)
\end{array}\right)\left(\begin{array}{c}
y_{n-1} \\
y_{n}
\end{array}\right) \\
& +0\left(M \epsilon(x)^{3}\right) .
\end{aligned}
$$

By Lemma 3, we conclude that, after a finite time $T_{n-1}^{\prime} \geq$ $T_{n-1}$, the solution satisfies

$$
\forall t \geq T_{n-1}^{\prime}: \quad\left\|\left(x_{n}, y_{n-1}\right)\right\|=0\left(M \epsilon(x)^{3}\right) .
$$

The above argument can be recursively repeated for each equation to prove that, after a finite time $T_{1}^{\prime} \geq T_{2}^{\prime} \geq \cdots \geq T_{n}^{\prime}$, the control law is no longer saturated and the closed-loop system has the form $\dot{y}=(A+0(M)) y$, where the matrix $A$ is a lower triangular filled with -1 . For $T \geq T_{1}^{\prime}$, the solution converges exponentially to the equilibrium $x=0$, which ends the proof.

Example 1: The system

$$
\begin{aligned}
& \dot{x}_{1}=x_{2}+x_{1}^{2} u^{2} \\
& \dot{x}_{2}=u+x_{1} u^{2}
\end{aligned}
$$

is homogeneous of degree one with respect to the dilation $\delta(x, u)=\left(\epsilon^{-1} x_{1}, x_{2}, \epsilon u\right)$ and therefore nonpeaking. The "radius" is in this case

$$
\rho(x)=\frac{1}{\sqrt{1+x_{1}^{2}}}
$$

and global asymptotic stability of the equilibrium $x=0$ is achieved by a control law of the form

$$
u=-\operatorname{sat}_{M \epsilon(x)}\left(x_{2}\right)-\operatorname{sat}_{M \epsilon(x)^{2}}\left(x_{1}+x_{2}\right) .
$$

The same control law multiplied by the gain $\gamma\left(x_{2}\right)=\min (1$, $\left.1 /\left(1+\left|x_{2}\right|^{q}\right)\right)$ can be employed to stabilize the system

$$
\begin{aligned}
& \dot{x}_{1}=x_{2}+x_{1}^{2} u^{2} \\
& \dot{x}_{2}=u+x_{2}^{q} x_{1} u^{2} .
\end{aligned}
$$

\section{A CASCADE Result}

The stabilization result for the system (1.3) is now extended to a more general class of nonlinear cascades

$$
\begin{aligned}
\left(\begin{array}{c}
\dot{x}_{1} \\
\vdots \\
\dot{x}_{n-1} \\
\dot{x}_{n}
\end{array}\right) & =\left(\begin{array}{c}
x_{2} \\
\vdots \\
x_{n} \\
c^{T} v
\end{array}\right)+P(x, v) v, \quad P(x, 0)=0 \\
\dot{\xi} & =a(\xi, u) \quad \xi \in \mathbb{R}^{p}, u \in \mathbb{R}, v=(\xi, u)^{T}
\end{aligned}
$$

for which we make the following assumption.

Assumption 2: The equilibrium $\xi=0$ of $\dot{\xi}=a(\xi, 0)$ is GAS and LES. The Jacobian linearization of (5.30) is stabilizable.

If the matrix $P(x, v)$ has a feedforward structure characterized by the condition that the $i$ th row does not depend on the variables $\left(x_{1}, \cdots, x_{i}\right)$, for $1 \leq i \leq n$, then the cascade (5.30) belongs to the class of (strict) feedforward systems and Assumption 2 is sufficient to derive global stabilization results (see [20], [7], and [13]). Under Assumption 2, indeed a unique linear change of coordinates $y=T x+S \xi$, exists with $T$ upper triangular, such that, in the new coordinates $(y, \xi)$ and with the linear feedback $u=-\sum_{i=1}^{n} y_{i}$, the Jacobian linearization of (5.30) becomes

$$
\begin{aligned}
\dot{y}_{1} & =-y_{1} \\
\dot{y}_{2} & =-y_{1}-y_{2} \\
& \vdots \\
\dot{y}_{n} & =-\sum_{i=1}^{n} y_{i} \\
\dot{\xi} & =A \xi-b \sum_{i=1}^{n} y_{i} .
\end{aligned}
$$

With a proper choice of the saturation constants, global stabilization of (5.30) is then achieved with the nested saturated feedback (2.7).

Here, we will not impose any structural condition on $P(x, u)$, but instead we restrict its growth like in the previous section.

Assumption 3: Each column $p_{i}(x, v)$ of the matrix $P(x, v)$ is at least of order zero with respect to the dilation $\delta_{\epsilon}(x, v)=$ $\left(\delta_{\epsilon}(x), \epsilon v\right)$, where $\delta_{\epsilon}(x)$ is the slow-peaking dilation (3.12).

Assumption 4: Two class- $\mathcal{K}$ functions $\chi_{1}$ and $\chi_{2}$ exist such that

$$
\|P(x, v)\| \leq \chi_{1}(\|v\|)\|x\|+\chi_{2}(\|v\|)
$$


guarantees that, whenever $u(t)$ is selected such that $\xi(t)$ exists for all $t \geq 0$, then $x(t)$ also exists for all $t \geq 0$ because

$$
\frac{d}{d t}(\|x(t)\|) \leq \chi_{3}(\|v(t)\|)\|x(t)\|+\chi_{4}(\|v(t)\|)
$$

for some class- $\mathcal{K}$ functions $\chi_{3}$ and $\chi_{4}$.

The additional growth Assumption 4 was not present in the previous section because the initial value of $u$ could be freely assigned. This result is in contrast to the initial value of the vector $v=(\xi, u)^{T}$, which now depends on the initial condition $\xi(0)$. Assumption 4 is necessary to prevent a finite escape time of $x(t)$ during the convergence of $\xi(t)$ to a sufficiently small neighborhood of the origin. This result is so even if the convergence of $\xi(t)$ can be rendered arbitrarily fast (a consequence of the fast peaking phenomenon; see [14]).

Theorem 2: Under Assumptions 2-4, low gains $\gamma_{2}\left(y_{n}\right)>0$ and $\bar{\epsilon}\left(y_{n}\right)>0$ and a constant $\bar{M}>0$ exist such that for all $M \in[0, \bar{M}]$, the low-gain control law

$$
\begin{aligned}
u= & -\gamma_{1}(\|\xi\|) \gamma_{2}\left(y_{n}\right)\left(\operatorname{sat}_{M \epsilon(x)}\left(y_{n}\right)\right. \\
& \left.+\operatorname{sat}_{M \epsilon^{2}(x)} \cdots+\operatorname{sat}_{M \epsilon^{n}(x)}\left(y_{1}\right)\right)
\end{aligned}
$$

achieves global asymptotic stability of the equilibrium $(x, \xi)=$ 0 of (5.30).

Proof: Because the equilibrium $\xi=0$ of $\dot{\xi}=a(\xi, 0)$ is LES, strictly positive constants $\alpha, \beta, \delta$ exist such that

$$
\|\xi\| \leq \delta \Rightarrow \frac{d}{d t}\|\xi\| \leq-\alpha \xi+\beta|u| .
$$

The ball $B(0, \delta)=\{\xi \mid\|\xi\| \leq \delta\}$ is clearly invariant if $|u| \leq$ $\bar{u}:=\beta^{-1} \alpha \delta$. Moreover, because the equilibrium $\xi=0$ of $\dot{\xi}=a(\xi, 0)$ is GAS, a nonlinear gain $\gamma_{1}(\|\xi\|)$ exists such that $\gamma_{1}(s)=1$ for $s \in[0, \delta]$ and such that all solutions of $\dot{\xi}=$ $a\left(\xi, \gamma_{1}(\|\xi\|) w\right)$ are attracted in finite time to the set $B_{\delta}$ provided that $|w| \leq(\bar{u} / 2)$. For any choice of $u=\gamma_{1}(|\xi| \mid) w$ that satisfies these constraints, $\xi(t)$ exists for all $t \geq 0$. By Assumption 4, the solutions of (5.30) then exist for all $t \geq 0$. In addition, for each initial condition $(x(0), \xi(0))$, a finite time $T_{\xi}>0$ exists such that $\xi(t) \in B_{\delta}$ for all $t \geq T_{\xi}$.

Let $\psi(t):=\gamma_{2}\left(y_{n}(t) \bar{\epsilon} \epsilon(x(t))\right.$. For $t \geq T_{\xi}$, we have $|u(t)| \leq$ $2 M \psi(t)$ and we show that a finite time $T_{u} \geq T_{\xi}$ exists such that

$$
\left\|\xi\left(T_{u}\right)\right\| \leq \frac{4 M \beta}{\alpha} \psi\left(T_{u}\right) .
$$

By contradiction, suppose that

$$
\forall t \geq T_{\xi}: \quad(\beta|u(t)| \leq) 2 M \beta \psi(t) \leq \frac{\alpha}{2}\|\xi(t)\| .
$$

Then, $(d / d t)\|\xi\| \leq-(\alpha / 2) \xi$ and the convergence of $v(t)=$ $(\xi(t), u(t))$ is exponential

$$
\|v(t)\|=\|(\xi(t), u(t))\| \leq C e^{-(\alpha / 2) t} .
$$

From (5.34) and (5.35), $\psi(t)$ also converges exponentially, which implies that at least one state $x_{i}(t), i \in\{1, \cdots, n\}$, diverges exponentially to infinity; that is, strictly positive constants $c_{1}$ and $c_{2}$ exist such that

$$
\left|x_{i}(t)\right| \geq c_{1} e^{c_{2} t} \text {. }
$$

Denoting by $e_{n}$ the vector $e_{n}=(0, \cdots, 0,1)^{T}$ and by $Q$ the matrix filled with zeros except for the superdiagonal elements $q_{i+1}=1$, we have

$$
\dot{x}=Q x+P(x, v) v+c^{T} v e_{n} .
$$

The matrix $Q$ has all its eigenvalues on the imaginary axis, so that a matrix $S>0$ exists, which satisfies the Lyapunov inequality $S Q+Q^{T} S \leq c_{2} S$. Using Assumption 4, the norm $\|x\|_{S}=\sqrt{x^{T} S x}$ satisfies for some constant $c_{3}>0$ and for $\|x\|_{S} \geq 1$

$$
\frac{d}{d t}\|x\|_{S} \leq \frac{c_{2}}{2}\|x\|_{S}+c_{3}\left(\chi_{1}(\|v\|)+\chi_{2}(\|v\|)+\|v\|\right)\|x\|_{S} .
$$

For $t$ sufficiently large, $\|v(t)\|$ is small enough such that

$$
\frac{d}{d t}\|x\|_{S} \leq \frac{3 c_{2}}{4}\|x\|_{S}
$$

which contradicts (5.36). We conclude that (5.33) holds.

Defining $z(t):=\|\xi(t)\|-(4 M \beta / \alpha) \psi(t)$, we have from (5.33) that $z\left(T_{u}\right) \leq 0$ and we will now show that $z(t) \leq 0$ for all $t \geq T_{u}$ by proving that

$$
z(t)=0 \Rightarrow \dot{z}(t) \leq 0 \text {. }
$$

An explicit calculation yields

$$
\begin{aligned}
z=0 \Rightarrow \dot{z} & =\frac{d}{d t}\|\xi\|-\frac{4 M \beta}{\alpha} \dot{\psi} \\
& \leq M \beta\left(-\gamma_{2}\left(y_{n}\right) \epsilon(x)\right)-\frac{4}{\alpha}\left(\left|\gamma_{2}^{\prime}\left(y_{n}\right) \dot{y}_{n} \epsilon(x)\right|+|\gamma \dot{\epsilon}|\right)
\end{aligned}
$$

If $z \leq 0$, then $\|\xi\|=0\left(\gamma_{2} M \epsilon\right)$ and $\dot{y}_{n}=0\left(\gamma_{2} M \epsilon\right)$. By Lemma 2, we conclude that $|\dot{\epsilon}| \leq \mu \epsilon$ with $\mu$ a positive constant, which can be selected arbitrarily small by a suitable choice of $\bar{\epsilon}\left(y_{n}\right)$. Also, $\gamma_{2}$ can always be chosen to satisfy $\left|\gamma_{2}^{\prime}\left(y_{n}\right)\right| \leq \mu$. We conclude

$$
z=0 \Rightarrow \dot{z} \leq-M \beta \gamma_{2}\left(y_{n}\right) \epsilon(x)\left(1-\frac{8 \mu}{\alpha}\right)
$$

so that (5.37) holds if $\mu$ is chosen sufficiently small.

Having established that $\|v\|=0(M \epsilon)$ for all $t \geq T_{u}$, the proof can be pursued exactly as in Theorem 1 to show the convergence of each solution to zero, starting with

$$
\dot{y}_{n}=-\gamma_{2}\left(y_{n}\right)\left(\operatorname{sat}_{M \epsilon(x)}\left(y_{n}\right)\right)+0\left(M \epsilon^{2}(x)\right)+P_{n}(x, v) v
$$

and adding a new equation at each step.

As an illustration of Theorem 2, we design a globally stabilizing control law for the celebrated frictionless ball-and-beam model [5].

Example 2: After a preliminary feedback, the ball-and-beam model considered in [5] is

$$
\begin{aligned}
& \dot{z}_{1}=z_{2} \\
& \dot{z}_{2}=-\sin z_{3}+z_{1} z_{4}^{2} \\
& \dot{z}_{3}=z_{4} \\
& \dot{z}_{4}=w
\end{aligned}
$$

where $z_{1}$ and $z_{2}$ denote the position and velocity of the ball and $z_{3}$ and $z_{4}$ denote the angular position and angular velocity of the beam. The term $-\sin z_{3}$ is because of gravity, and the term $z_{1} z_{4}^{2}$ is the centrifugal acceleration of the ball because of the motion of the beam. To apply Theorem 2, we rewrite (5.38) as

$$
\begin{aligned}
& \dot{x}_{1}=x_{2} \\
& \dot{x}_{2}=\xi_{1}+\left(\sin \xi_{1}-\xi_{1}\right)+x_{1} \xi_{2}^{2} \\
& \dot{\xi}_{1}=\xi_{2} \\
& \dot{\xi}_{2}=-w:=-k_{1} \xi_{1}-k_{2} \xi_{2}+u, \quad k_{1}>0, k_{2}>0 .
\end{aligned}
$$


Because of the nonlinearity $x_{1} \xi_{2}^{2}$, the system (5.39) is not in the feedforward form required for a forwarding design. The growth conditions of Theorem 2, however, are satisfied: the slow-peaking dilation is

$$
\delta_{\epsilon}(x, \xi, u)=\left(\epsilon^{-1} x_{1}, x_{2}, \epsilon \xi_{1}, \epsilon \xi_{2}, \epsilon u\right)
$$

and the radius is $\rho(x)=\left(1 / \sqrt{1+x_{1}^{2}}\right)$. The perturbation $\left(0,\left(\sin \xi_{1}-\xi_{1}\right)+x_{1} \xi_{2}^{2}\right)^{T}$ is of order one. Also, the linear growth in $x$, that is, Assumption 4 , is satisfied.

Using the change of coordinates

$$
\begin{aligned}
& y_{1}=k_{1} x_{1}+\left(k_{1}+k_{2}\right) x_{2}+\left(1+k_{2}\right) \xi_{1}+\xi_{2} \\
& y_{2}=k_{1} x_{2}+k_{2} \xi_{1}+\xi_{2}
\end{aligned}
$$

GAS of the equilibrium $z=0$ is achieved with a control law of the form

$$
u=-\gamma_{2}\left(y_{2}\right)\left(\operatorname{sat}_{M \epsilon(x)}\left(y_{2}\right)-\operatorname{sat}_{M \epsilon(x)^{2}}\left(y_{1}\right)\right) .
$$

The gain $\gamma_{1}(\|\xi\|)$ used in Theorem 2 is not necessary because the $\xi$-subsystem is linear.

\section{CONCLUSION}

In this paper, we have shown that semiglobal and global stabilization of a chain of integrators perturbed by a vector field, which is higher order in $u$ cannot be guaranteed without extra conditions on the perturbation, despite the fact that the unperturbed chain of integrators can be stabilized by using a low-gain design. The obstacle to large regions of attractions for the perturbed system is caused by the large state transients inherent to the low-gain design, a phenomenon that we call slow peaking in contrast to the fast-peaking phenomenon associated with high-gain designs [17]. To overcome the destabilizing effect of peaking, we must impose growth conditions on the nonlinearities. We have characterized these growth conditions as higher order conditions with respect to a weighted dilation, in which each state is weighted by its peaking exponent. When this higher order condition is satisfied, we have shown that global stabilization of the perturbed system can be achieved by a low-gain design that preserves the higher order property in closed loop. This global stabilization result has been extended to the case when the perturbed chain of integrators is cascaded with a GAS/LES subsystem.

\section{APPENDIX}

\section{A. Proof of Lemma 1}

The proof goes by contradiction. Let $i \in\{1, \cdots, n\}$, and let $x(0)$ such that $x_{k}(t) \leq \bar{x}_{k}$ for all $t \geq 0$ and $i \leq k \leq n$. Assume that, for this initial condition, the integral (4.22) is bounded; that is, the time function $f(t)=\rho(x(t))^{n-i+1}$ belongs to $L^{1}(0, \infty)$. Because $f(t)$ is uniformly continuous, we have $f(t) \leq(C / t)$ for some constant $C$.

Because $p_{k}(x, u)$ is of order $k-n$ and $u=k(x)$ is of order $n-i+1$, we deduce from Proposition 2 that

$$
k(x) p_{k}(x, k(x))=0\left(\rho(x)^{n-2 i+k+1}\right)
$$

so that, for $1 \leq k \leq i-1$, we have

$$
\dot{x}_{k}=x_{k+1}+h_{k}(x), \quad h_{k}(x)=0\left(\rho^{n-2 i+k+1}\right) .
$$

The time function $h_{k}(x(t))$ satisfies

$$
\left|h_{k}(x(t))\right| \leq \gamma f(t) \rho(x(t))^{k-i} \leq \gamma \frac{C}{t} \rho(x(t))^{k-i} .
$$

Because $k-i<0$ and $\rho(x(t))<1$, we have that $\rho^{k-i}<$ $\left(\rho^{n-i+1}\right)^{k-i}$, and therefore we conclude that

$$
\left|h_{k}(x(t))\right| \leq \gamma \frac{C}{t}(f(t))^{k-i} \leq C^{\prime} t^{i-1-k}
$$

for some constant $C^{\prime}>0$. Subsituting in (8.40), we obtain

$$
\left|\dot{x}_{k}\right| \leq\left|x_{k+1}\right|+C^{\prime} t^{i-1-k}, \quad 1 \leq k \leq i-1 .
$$

Using the fact that $x_{i}(t)$ is bounded for all $t \geq 0$, we obtain by integration the estimates

$$
\left|x_{k}(t)\right| \leq K t^{i-k}, \quad 1 \leq k \leq i-1
$$

for some constant $K>0$. Substituting the estimates (8.41) in $\rho(x)$, we conclude

$$
\begin{aligned}
f(t)= & \left(1+\sum_{k=1}^{n-1} \alpha_{k} x_{k}(t)^{(2(n-1)) /(n-k)}\right)^{-((n-i+1) /(2(n-1)))} \\
\geq & \left(1+\sum_{k=i}^{n} \alpha_{k} \bar{x}_{k}^{(2(n-1)) /(n-k)}\right. \\
& \left.+\sum_{k=1}^{i-1} \alpha_{k}(K t)^{(2(n-1)(i-k)) /(n-k)}\right)^{-((n-i+1) /(2(n-1)))} \\
\geq & \frac{1}{C_{1}+C_{2} t}
\end{aligned}
$$

for some constants $C_{1}>0$ and $C_{2}>0$, which contradicts the assumption that $f \in L^{1}(0, \infty)$.

\section{B. Proof of Lemma 2}

Because $\rho(x)$ is a function of order one and $f(x, u)$ is a vector field of order one, the time derivative $\dot{\rho}=L_{f} \rho$ is of order two. By Proposition 2, a function $C\left(x_{n}\right)>0$ exists such that

$$
|u| \leq \rho(x) \Rightarrow|\dot{\rho}(x)| \leq C\left(x_{n}\right) \rho^{2}(x) .
$$

Choose a smooth and strictly decreasing function $\kappa(s)>0$ such that $C\left(x_{n}\right) \kappa\left(\left|x_{n}\right|\right) \leq \mu$ for all $x_{n} \in \mathbb{R}$. Because $\kappa^{\prime}(s) \rightarrow 0$ as $s \rightarrow \infty$, a constant $\bar{s}$ large enough exists such that $\left|\kappa^{\prime}(s)\right| \leq$ $\mu / K_{u}$ for all $s \geq \bar{s}$. We then choose

$$
\begin{aligned}
\bar{\epsilon}\left(x_{n}\right) & =\kappa(\bar{s}), \quad\left|x_{n}\right| \leq \bar{s} \\
& =\kappa\left(\left|x_{n}\right|\right), \quad\left|x_{n}\right| \geq \bar{s}
\end{aligned}
$$

so that $\left|\bar{\epsilon}^{\prime}\left(x_{n}\right)\right| \leq \mu$ for all $x_{n}$. With this choice, we obtain

$$
\epsilon(x)=\bar{\epsilon}\left(x_{n}\right) \Rightarrow \dot{\epsilon} \leq \bar{\epsilon}^{\prime}\left(x_{n}\right) \dot{x}_{n} \leq \mu \epsilon(x)
$$

and

$$
\epsilon(x)=\rho(x) \Rightarrow \dot{\epsilon}=\dot{\rho} \leq C\left(x_{n}\right) \rho^{2} \leq C\left(x_{n}\right) \bar{\epsilon}\left(x_{n}\right) \epsilon \leq \mu \epsilon(x)
$$

which proves the lemma.

\section{Proof of Lemma 3}

Because $A$ is Hurwitz, constants $\alpha$ and $\beta>0$ exist such that

$$
\frac{d}{d t}\|x(t)\| \leq-\alpha\|x(t)\|+\beta \epsilon(t) .
$$


Define $z(t)=\alpha\|x(t)\|-2 \beta \epsilon(t)$. If $z(t)>0$ for all $t \geq 0$, then $\|x(t)\| \geq(2 \beta / \alpha) \epsilon(t)$ and $(d / d t)\|x(t)\| \leq-(\alpha / 2)\|x(t)\|$ for all $t \geq 0$. Then, we have

$$
\forall t \geq 0: \quad \epsilon(0) e^{-\mu t} \leq \epsilon(t) \leq K\|x(t)\| \leq K\|x(0)\| e^{-(\alpha / 2) t}
$$

which is a contradiction for $t$ large enough if $\mu<(\alpha / 2)$. Hence, a finite time $T \geq 0$ exists such that $z(T) \leq 0$. We then show that $z(t) \leq 0$ for all $t \geq T$ by proving

$$
z(t)=0 \Rightarrow \dot{z}(t) \leq 0 .
$$

An explicit calculation yields

$$
z(t)=0 \Rightarrow \dot{z} \leq-\beta \epsilon(t)-2 \frac{\beta}{\alpha} \dot{\epsilon} \leq-\beta\left(1-\frac{2 \mu}{\alpha}\right) \epsilon
$$

so that (8.42) holds if $\mu$ is small enough.

\section{ACKNOWLEDGMENT}

The author gratefully acknowledges P. Kokotović for numerous discussions about peaking and for his comments about a preliminary version of this paper. This paper presents research results of the Belgian Programme on Interuniversity Poles of Attraction, initiated by the Belgian State, Prime Minister's Office for Science, Technology and Culture. The scientific responsibility rests with the author.

\section{REFERENCES}

[1] C. Barbu, R. Sepulchre, P. V. Kokotovic, and W. Lin, "Global asymptotic stabilization of the ball-and-beam model," in Proc. 36th Conf. Decision Contr., 1997, pp. 2351-2355.

[2] J. H. Braslavsky and R. H. Middleton, "Global and semi-global stabilizability in certain cascade nonlinear systems," IEEE Trans. Automat. Contr., vol. 41, pp. 876-880, 1996.

[3] J.-M. Coron, L. Praly, and A. Teel, "Feedback stabilization of nonlinear systems: Sufficient conditions and Lyapunov and input-output techniques. Minicourse on stabilization of nonlinear system," in Trends in Control, A. Isidori, Ed. Berlin: Springer-Verlag, 1995, pp. 293-347.

[4] F. Grognard, R. Sepulchre, G. Bastin, and L. Praly, "Nested linear low-gain designs for semiglobal stabilization of feedforward systems," in Proc. 4th NOLCOS IFAC Symp., Enschede, 1998, pp. 829-834.

[5] J. Hauser, S. Sastry, and P. V. Kokotović, "Nonlinear control via approximate input-output linearization: The ball and beam example," IEEE Trans. Automat. Contr., vol. 37, pp. 392-398, Mar. 1992.

[6] H. Hermes, "Nilpotent and high order approximations of vector field systems," SIAM Rev., vol. 33, pp. 238-264, 1991.

[7] M. Jankovic, R. Sepulchre, and P. V. Kokotović, "Constructive Lyapunov stabilization of nonlinear cascade systems," IEEE Trans. Automat. Contr., vol. 41, pp. 1723-1735, 1996.

[8] M. Kawski, "High-order local controllability," in Nonlinear Controllability and Optimal Control, H. J. Sussmann, Ed. New York, NY: Academic, 1990, vol. 133, pp. 431-467. Pure and Applied Mathematics.
[9] P. V. Kokotović and R. Marino, "On vanishing stability regions in nonlinear systems with high-gain feedback," IEEE Trans. Automat. Contr. vol. 31, pp. 967-970, 1986

[10] M. Krstić, I. Kanellakopoulos, and P. V. Kokotović, Nonlinear and Adaptive Control Design, New York: Wiley, 1995.

[11] Z. Lin and A. Saberi, "Semi-global stabilization of partially linear composite systems via feedback of the state of the linear part," Syst. Contr. Lett., vol. 20, pp. 199-207, 1993.

[12] — - "Semiglobal exponential stabilization of linear systems subject to input saturation via linear feedback," Syst. Contr. Lett., vol. 21, pp. 225-239, 1993.

[13] F. Mazenc and L. Praly, "Adding an integration and global asymptotic stabilization of feedforward systems," IEEE Trans. Automat. Contr., vol. 41, pp. 1559-1578, 1996.

[14] A. Saberi, P. V. Kokotović, and H. J. Sussmann, "Global stabilization of partially linear composite systems," SIAM J. Contr. Optim., vol. 28, pp. 1491-1503, 1990.

[15] R. Sepulchre, M. Jankovic, and P. V. Kokotović, Constructive Nonlinear Control. New York, NY: Springer-Verlag, 1997.

[16] E. D. Sontag, "Remarks on stabilization and input-to-state stability," in Proc. 28th CDC, Tampa, FL, 1989, pp. 1376-1378.

[17] H. J. Sussmann and P. V. Kokotović, "The peaking phenonmenon and the global stabilization of nonlinear systems," IEEE Trans. Automat. Contr, vol. 36, pp. 424-439, 1991

[18] H. J. Sussmann, E. D. Sontag, and Y. Yang, "A general result on the stabilization of linear systems using bounded controls," IEEE Trans. Automat. Contr., vol. 39, pp. 2411-2425, 1994.

[19] A. R. Teel, "Global stabilization and restricted tracking for multiple integrators with bounded controls," Syst. Contr. Lett., vol. 18, pp. 165-171, 1992

[20] A. Teel, "Using saturation to stabilize a class of single-input partially linear composite systems," in Proc. IFAC NOLCOS'92 Symp., Bordeaux, June 1992.

[21] _ _ "Semiglobal stabilizability of linear null controllable systems with input nonlinearities," IEEE Trans. Automat. Contr., vol. 40, pp. 96-100, 1995.

[22] A. R. Teel and L. Praly, "Tools for semiglobal stabilization by partial state feedback and output feedback," SIAM J. Contr. Optim., vol. 33, pp. 1443-1488, 1995

[23] A. Teel, "A nonlinear small gain theorem for the analysis of control systems with saturation," IEEE Trans. Automat. Contr., vol. 41, pp. 1256-1270, 1996

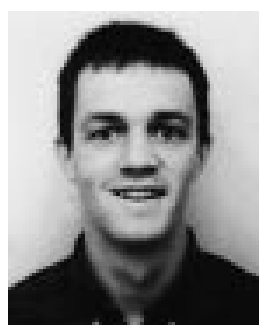

Rodolphe Sepulchre (M'96) was born in Brussels, Belgium, in 1967. He received the University and Ph.D. degrees in engineering science (applied mathematics) from the University of Louvain in 1990 and 1994, respectively.

From 1994 to 1996, he was a Postodoctoral Research Fellow at the Center for Control Engineering and Computation, at the University of California, Santa Barbara. He is currently an Associate Professor at the University of Liege, Belgium. His research interests lie in control theory, nonlinear dynamical systems, and their applications to problems in mechanical, electrical, and biomedical engineering.

He has coauthored with M. Jankovic and P. Kokotovic, the book "Constructive Nonlinear Control" (Springer, 1997). 